\title{
Internet of Things Application to Monitoring Plant Disease and Insect Pests
}

\author{
Yun Shi, Zhen Wang, Xianfeng Wang, Shanwen Zhang \\ XiJing University, Xi'an Shanxi 710123, China \\ wjdw716@163.com
}

Key words:Internet of things (IOT); disease and insect pest; Sensor; Agricultural information

\begin{abstract}
Usinginformationand communicatingtechnologyestablish plantdisease andinsect pests long-distance monitoring system is an effective way for improve low technical culture quality of the farmers, since the expert unable to go to the farm to manage the agriculture disease and insect pests. This paper introduces the concept of internet of things (IOT) technology topercept information, and discusses the role of the IOT technology in agricultural disease and insect pest control, which includes agricultural disease and insect pest monitoring system, collecting disease and insect pest information using sensor nodes, data processing and mining, etc.A disease and insect pest control system based on IOTis proposed, which consisted of three levels andthree systems. The system can provide a new way to access agricultural information for the farm.
\end{abstract}

\section{Introduction}

Plant disease and insect pests have turned into a dilemma as it can causesignificant reduction in both quality and quantity ofagricultural products [1,2]. China is one of the countries which suffer from the most serious plant disease and insect pest infection in the world. In recent decades, the loss caused by plant disease and insect pests is far more severe than that by plant fires, so plant disease and insect pests forecasting is of great significance and quite necessary. The naked eye observation of experts is the main approachadopted in practice for detection and identification of plantdisease and insect pests [3]. However, this requires continuous monitoring ofexperts which might be prohibitively expensive in large farms.Further, in some developing countries, farmers may have to golong distances to contact experts, whichtakethe expertstoo expensive and time consuming [4].Automatic detection of plant diseases is an essential researchtopic as it may prove benefits in monitoring large fields ofcrops, and thus automatically detect the symptoms of disease and insect pestsas soon as they appear on plant leaves [2-4]. Machine learning based on detection and recognition of plantdisease and insect pests can provide clues to identify and treat the plant disease and insect pests inits early stages [5]. But, machine learning is only a process in the recognition methods. Looking for fast, automatic, less expensive and accurate methodto detect plant disease and insect pest cases is of great realistic significance. Recently, Internet of things (IOT) is a hottest topic, and many researchers have begun studying how to use IOT in agriculture [6,7].IOT would be a great breakthrough in modern agriculture field. Many kinds of various sensors have been produced for sensing agricultural objects, such as crops, animals, at thesame time the sensed data can be transmitted to Internet by wireless network.IOT providesa change for the remote monitoring the plant disease and insect pests. Its main techniques such as RFID, sensor and 3S have already been applied in a certain scale [8]. At present, in many developed countries such as America, Holland, Japan and European Union, the research of agriculture IOT has gotten a very high level. With the development of IOT, especially the development of IOT technology (such as RFID, Sensor etc.) provides new opportunity for research on plant disease and insect pests automatically recognition and detection [10].Infomationization, internetization and intelligentization of agricultural IOT would be a new researching content of agricultural modernization and trend of global agriculture development. The repaid development of agricultural IOT has an important influence on realizing intensive agriculture, high yield and high quality, and it will provide a solid foundation for the development of agriculture information technologies. 


\section{Data acquisition equipment in IOT}

According to the characteristic of agricultural information flow, from the side of technology, because of the attributes of overall sense, reliable transfer and intelligent process, IOT begins to become the main method of data acquisition and transmission and would become an important technology across several kinds of sensors to collect, analyze, transmit and manage the whole data related to plant disease and insect pests.The sensor is an important technology for data acquisition, and is mainly used to capture part of the existing data, correlate and synchronize these data, analyze them, and finally, carries out a reactive activity without user intervention. The components of a (remote) sensing node include the following: sensing and actuation unit (single element or array), processing unit, communication unit, power unit and other application-dependent units Sensors can be simple point elements orcan be multipoint detection arrays, and it has the capability of large scale deployment, low maintenance, scalability, adaptability for different scenarios etc.[9]. The technology of $3 \mathrm{~S}$ is mainly used to provide the location of a particular tagged object, acquire the information of an object or phenomenon by multiple satellites, aircraft, etc., and finally store, analyze, manage and present data that are linked to location(s) by GIS software[10]. For the transmission of information, wireless sensor network (WSN) and GPRS/GSM are the mainly used network technology in the field of agriculture. WSN mainly consists of spatially distributed autonomous sensors to cooperatively monitor physical or environmental conditions, such as temperature, sound, pressure, motion and pollutants, etc. Agricultural monitor of disease and insect pests is a very complicated process, and involves many different phases and different actors. Every phase involves many kinds of operation, and every operation involves many technologies, includes data collection, image processing, data mining and fusion, pattern recognition, etc. Among of them, automatic data collection module mainly includes, wind speed and direction, air temperature and humidity, carbon dioxide concentration, soil temperature and humidity, conductivity and video information; wireless transmission network module is responsible for the collected information quickly and efficiently and reliably to the total node; remote data transmission module is responsible for the summary of monitoring data points through the GPRS network by Internet transfer to the database; the main function of the data management module is stored in the attribute data, data entry, delete, modify, storage, retrieval and statistics etc. The data are the core in monitoring system based onIOT. The potential of using these data will reach its full extent when suitable collecting and transmitting technology and method are developed.

\section{Architecture of IOT in agricultural monitoring system}

In the monitoring system of agricultural disease and insect pests, the main aim is to achieve the disease and insect pests monitoring information and collection of the IOT. Then the design and selection of the node will be the key to the whole monitoring system. From the architecture of the agricultural disease and insect pests, the overall structure of the monitoring information system point of viewis divided into four categories: network sensor node, acquisition of monitoring information, sensor and wireless communication technology, intelligent data processing. The system consists of sensor nodes, communication system, Internet, information service terminal and monitoring software and hardware system, and the system structure is shown in Fig. 1.Sensor nodes are laid on targeted farmland areas and collected environmentalsignals are treated as a source of information. 


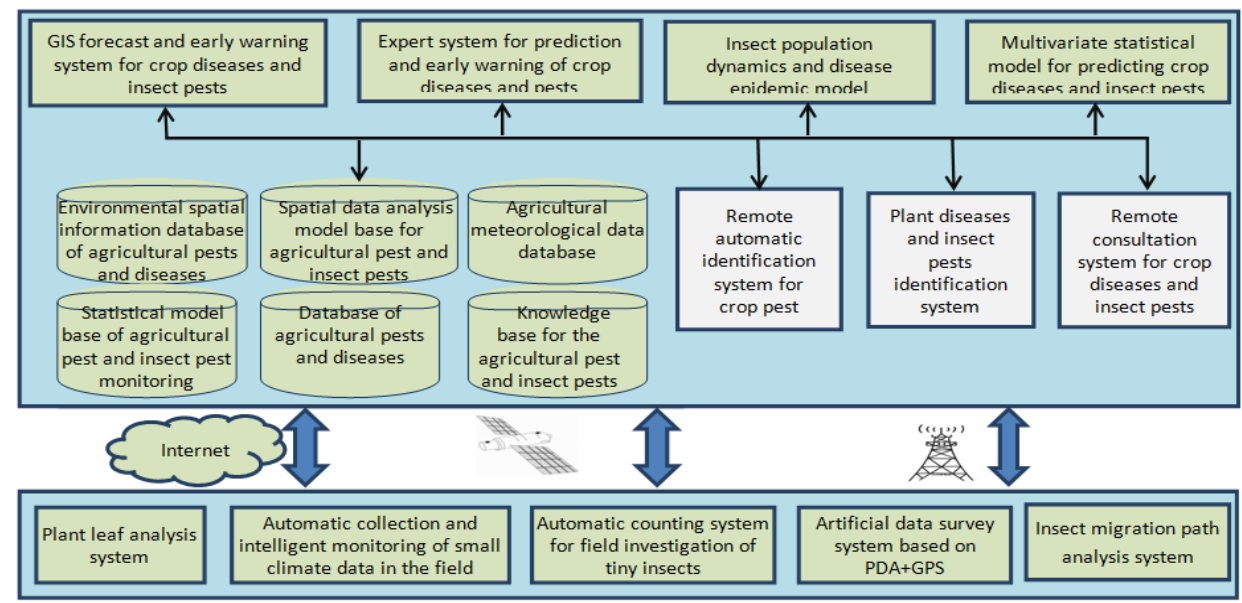

Fig.1Agricultural disease and insect pest disasters information monitoring system

\section{Disease and insect pest monitor platform}

The platform includes administrators, experts and ordinary visitors. Through computers, mobile phones, tablet computer, etc.,they can login the remote server and choose to view the real-time data, historical data and remote parameter settingcollected by the monitoring sensing layer; agricultural experts can analyze the collected data andconstruct an expert database.The information platform of disease and insect pests monitorbased on IOT would be an integrated system that integrates all kinds of information which includes the agricultural management knowledge, purchase, warehousing, delivery and retail and realizes the information interchange between different phases. The core function is fast acquisition, seamless connection, reliable transmission and in-time search and trackback of information (Fig.2).

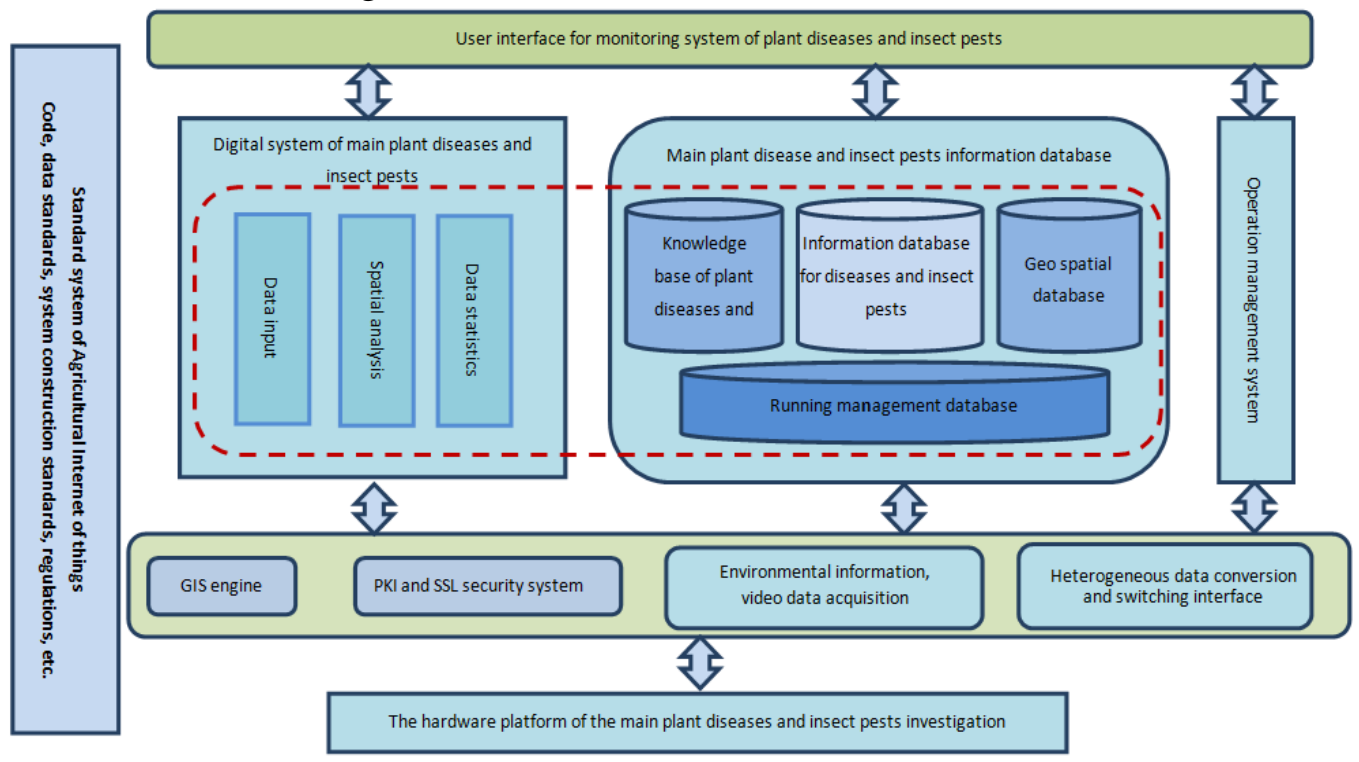

Fig.2 Interwork structure of IOT

\section{Conclusion}

The repaid development of IOT has an important influence on realizing intensive agriculture, high yield and high quality, and it will provide solid foundation for the development of agriculture information technologies. There is no doubt that efficient fresh agricultural products supply chain management is the key to improve the competitiveness of fresh produce enterprises, and the appearance of things brings a new opportunity for fresh agricultural products supply chain management. At present, there is still a big gap between theoretical research and practical applications, Especially in China, most of the literature of the IOT still remain in the introduction of the IOT itself and its application, but with the development of the relevant 
technology and the deepening of theoretical research and application, the IOT will play a positive role in the development of the fresh agricultural products supply chain in the future, it will also become an important direction of future research. So, the future of the IOT's application in fresh agricultural products supply chain management is bright, but the road is tortuous.

\section{Acknowledgement}

This work is partially supported by China National Natural Science Foundation under grant No. 61473237. It is also supported by the Shaanxi provincial education department Foundation under grant No. 2013JK1145 and Key cultivation project of Xijing University No.XJ13B02.

\section{References}

[1] H. Al-Hiary, S. Bani-Ahmad, M. Reyalat. Fast and Accurate Detection and Classification of Plant Diseases. International Journal of Computer Applications,2011,17(1):31-38.

[2] T. Rumpf, A. K. Mahlein, U. Steiner, et al. Early detection and classification of plant diseases with Support Vector Machines based on hyperspectral reflectance. Computers and Electronics in Agriculture,2010:74:91-99.

[3] H Al-Hiary, S Bani-Ahmad, M Reyalat, et al. Article: Fast and Accurate Detection and Classification of Plant Diseases. International Journal of Computer Applications,2011,17(1):31-38.

[4] Yin Laiwu, Chen Deyun, Li Changcheng, Chen Dong. A Novel Recognition Algorithm Based on Optimal Wavelet Packet and Non-Negative Matrix Factorization for Extracting Pathologic Features of Plant Image. International Journal of Signal Processing, Image Processing and Pattern Recognition,2013,6(5): 89-100

[5] Werner Allen G, Lorinez K, Ruiz M, et al.Deploying a wirelesssensor network on an active volcano. IEEE Internet Computing, 2006,10(2):18-25.

[6] Raghavendra V. Kulkarni, Anna Forster, Ganesh Kumar Venayagamoorthy.Computational Intelligence in Wireless Sensor Networks: A Survey. IEEECommunications Survey \& Tutorials,2011,13(1):1-29.

[7] Siquan Hu, Haiou Wang, Chundong She, Junfeng Wang. AgOnt: Ontology for Agriculture Internet of Things.IFIP Advances in Information and Communication Technology, 344,2011, 131-137.

[8] Welbourne E, Battle L, Cole G, et al. Building the Internet of Things using RFID. IEEE Internet Computing, 2009, 13(3):48-55.

[9] Thiago Teixeira, Sara Hachem, Valerie Issarny, NikolaosGeorgantas. ServiceOriented Middleware for the Internet of Things: A Perspective. Service Wave,2011,LNCS 6994:220-229.

[10] Luigi Atzori, Antonio Iera, GiacomoMorabito. The Internet of Things: A Survey.Computer Networks, 2010(54): 2787-2805 